\title{
Zeit und Geschichte in frühen Kulturen
}

\author{
Jan Assmann, Heidelberg
}

Zeit, wie wir sie gemeinhin erfahren, ist nicht die physikalisch gemessene, sondern die kulturell konstruierte Zeit. Uns sind nicht nur wie den Tieren Sinne gegeben, uns im Raum zu orientieren, sondern auch ein Gedächtnis zur Orientierung in der Zeit. Der Mensch verfügt über die Gabe, sich an Vergangenes zu erinnern und sich in die Zukunft zu entwerfen. In seinem Bewußtsein dehnen sich Erfahrungsräume und Erwartungshorizonte, die sogar über seine eigene Lebenszeit hinausgreifen. Welche Formen solche Erfahrungsräume und Erwartungshorizonte in einer gegebenen Gesellschaft ausbilden, hängt von der spezifischen Zeitkonstruktion dieser Gesellschaft ab. In solchen kulturellen Konstruktionen der Zeit prägen sich typischerweise zwei Dichotomien aus. Die eine betrifft die Unterscheidung zwischen einer Art kulturellem Lang- und Kurzzeitgedächtnis, die andere den Gegensatz von Reversibilität und Irreversibilität oder, um die hierfür gebräuchlichen geometrischen Metaphern zu verwenden, von Kreis und Pfeil, von Zyklizität und Linearität. In meinem Vortrag möchte ich zunächst im Sinne einer allgemeinen Einführung in die kulturelle Konstruktion der Zeit die Langzeit/Kurzzeit-Dichotomie und den Zusammenhang zwischen Zeitkonstruktion, Gedächtnis und Selbstbild beleuchten, um dann im Hauptteil speziell auf die altägyptische Zeitkonstruktion mit ihrer Unterscheidung des Zyklischen und des Linearen einzugehen.

\section{Langzeit- und Kurzzeitgedächtnis der Kultur}

Beginnen wir also mit der Unterscheidung von Kurz- und Langzeitgedächtnis. Das Ich, hat der Konstanzer Soziologe Thomas Luckmann einmal geschrieben, ist aus dem Stoff der Zeit gemacht, und zwar aus drei Arten von Zeit. Luckmann nennt sie die innere, die soziale und die historische Zeit. ${ }^{1}$ Die innere Zeit haben die Lebensphilosophen und Phänomenologen von Schopenhauer über Nietzsche und Bergson bis zu Husserl und William

1 Thomas Luckmann, „Remarks on Personal Identity: Inner, Social and Historical Time“, in: Anita Jacobson-Wedding, Identity: Personal and Socio-Cultural, Stockholm und Atlantic Highlands 1983, 67-91. 
James als inneres Zeitbewußtsein und Bewußtseinsstrom beschrieben, die soziale Zeit haben die Soziologen, Sozialpsychologen und Interaktionisten von Durkheim über Halbwachs und George Herbert Mead bis zu Erving Goffman als kommunikative Synchronisierung analysiert, die historische Zeit aber, also die Formen, in denen wir die weit über unsere Lebensspanne hinausgreifende, in Texten, Bildern und Riten symbolisch objektivierte Zeit in unser Selbstbild hineinnehmen und zum Stoff unserer Ich-Formation machen, beginnt erst in den letzten Jahren zum Gegenstand wissenschaftlicher Erforschung zu werden, und die Wissenschaft, die sich um diese Art von Zeit kümmert, ist nicht die Psychologie und die Soziologie, sondern sind die Kulturwissenschaften.

Der Begriff, unter dem diese Verbindung von Ich und Zeit verhandelt wird, ist das Gedächtnis. Entsprechend den drei Zeitarten, in denen Zeit zum Baustoff des Ichs wird, können wir von einem inneren oder personalen, einem sozialen oder kommunikativen und einem historischen Gedächtnis sprechen. Aber sowohl der Luckmannsche Begriff der „historischen Zeit“, als auch der entsprechende Begriff des historischen Gedächtnisses greifen zu kurz und legen die Gefahr nahe, das, worum es hier geht, mit so etwas wie Geschichtsbewußtsein zu verwechseln. Diese Art von Zeit, aus der sich ein menschliches Selbstbild und Ich-Bewußtsein aufbauen kann, tritt nämlich in verschiedenen kulturellen Konstruktionen auf, von denen die historische Zeit nur eine ist. Eine vollkommen andere Form von Zeitkonstruktion und Ich-Konstitution ist die mythische Zeit, die Thomas Mann in seinen Joseph-Romanen so unübertrefflich veranschaulicht hat. ${ }^{2}$ Daher sprechen wir, d.h. Aleida Assmann und ich, von „kultureller Zeit“ und vom „kulturellen Gedächtnis“ als der Form, uns in dieser Zeit zu orientieren und sie zum Stoff unserer Ich-Konstitution zu machen.

Die genannten drei Zeitformen, in denen sich das Ich als Gedächtnis konstituiert, der inneren, sozialen und kulturellen Zeit, lassen sich als drei Dimensionen des Gedächtnisses beschreiben: als neuronale, soziale und kulturelle Dimension. Um ein Gedächtnis auszubilden, genügt es nicht, ein Gehirn mit einer entsprechenden neuronalen Ausstattung zu haben; wie Maurice Halbwachs und in seinem Gefolge eine Fülle sozialpsychologischer Untersuchungen gezeigt haben, bedarf es zur Ausbildung eines individuellen Gedächtnisses darüber hinaus mindestens ebenso sehr der Sozialisa-

2 Verf., Thomas Mann und Ägypten: Mythos und Monotheismus in den Josephsromanen, München 2006. 
tion und Kommunikationen in verschiedenen gesellschaftlichen Gruppen. ${ }^{3}$ Halbwachs'These war, daß sich das Gedächtnis im Einzelnen genau wie die Sprache nur im Zuge seiner Sozialisation und Kommunikation aufbaut. Er nannte dies irreführenderweise das „kollektive“ Gedächtnis, so als handele es sich um das Gedächtnis von Gruppen im Gegensatz zu dem von Individuen; gemeint ist aber die Gesellschaft als eine Dimension des individuellen Gedächtnisses. Diese beiden Dimensionen sind heute unbestritten; wir können sie mit dem amerikanischen Psychoanalytiker und Soziologen Jeffrey Prager als „embodied“ und „embedded“ memory, also vielleicht „verkörpertes“ und „vernetztes Gedächtnis“ bezeichnen. ${ }^{4}$ Es handelt sich um zwei Dimensionen ein und desselben Gedächtnisses, unseres menschlichen je persönlichen Gedächtnisses, das einerseits eine Sache unserer Hirnzellen ist und in allen Sinnen und Fasern unseres Körpers steckt, und andererseits, wie das Bewußtsein überhaupt, sich erst in der Interaktion mit anderen aufbaut und entfaltet.

Aber auch in diesen beiden Dimensionen ist das Phänomen des menschlichen Gedächtnisses und damit der menschlichen Zeitkonstruktion und -orientierung noch nicht adäquat erfaßt. Aleida Assmann und ich arbeiten seit ca. 20 Jahren an der theoretischen Erschließung einer dritten Dimension: der kulturellen Dimension des Gedächtnisses. ${ }^{5}$ Unsere Erinnerungen sind nicht nur sozial, sondern auch kulturell „eingebettet“, wir gehen nicht

3 M. Halbwachs, Les Cadres sociaux de la mémoire, Paris 1925; Neuausgabe von G. Namer, Albin Michel 1994; La Mémoire collective, hg. von Jeanne Alexandre; kritische Neuausgabe: édition critique de G. Namer avec la collaboration de M. Jaisson, Albin Michel, Paris 1997 (Paris 1942, Neuauflage 1971); La Topographie légendaire des Evangiles en Terre sainte, dt. Stätten der Verkündigung im Heiligen Land, Konstanz 2003. Zur Gedächtnistheorie von Maurice Halbwachs s. zuletzt Hermann Krapoth, Denis Laborde (Hg.), Erinnerung und Gesellschaft. Mémoire et Société,Jb. für Soziologiegeschichte, Wiesbaden 2005. Über die neuronalen und sozialen Grundlagen des Gedächtnisses informiert Harald Welzer, Hans J. Markowitsch, Das autobiografische Gedächtnis. Hirnorganische Grundlagen und biosoziale Entwicklung, Stuttgart 2005. Speziell zum sozialen Gedächtnis s. Harald Welzer, Das kommunikative Gedächtnis, München 2002.

4 S.J. Prager, Presenting the Past. Psychoanalysis and the Sociology of Misremembering, Cambridge Mass. 1998.

5 J. Assmann, Das kulturelle Gedächtnis. Schrift, Erinnerung und politische Identität in frühen Hochkulturen, München 1992; Aleida Assmann, Erinnerungsräume. Formen und Wandlungen des kulturellen Gedächtnisses, München 1999, sowie unsere Beiträge in Erwägen, Wissen, Ethik 13,2 (2002), 183-247. Eine ausgezeichnete Zusammenfassung bei Astrid Erll, a.a.O., 27-33. 
nur mit anderen Personen, sondern auch mit Texten, Bildern, Dingen, Symbolen, Orten, Landschaften und Riten um. Für die kulturelle Konstruktion der Zeit nun verläuft die entscheidende Trennungslinie zwischen dem persönlichen und kommunikativen Gedächtnis auf der einen, und dem kulturellen Gedächtnis auf der anderen Seite, der Unterscheidung zwischen dem in lebendigen Gedächtnisträgern verkörperten Gedächtnis auf der einen, und dem in symbolische Formen investierten Gedächtnis auf der anderen Seite. Diese Differenz ist groß genug, um den Gedächtnischarakter der Kultur lange Zeit zu verschleiern und ihn noch heute vielen als eine inakzeptable Metaphorik wenn nicht gar Mystifikation erscheinen zu lassen. Daher sei betont, daß es sich hier nicht um eine Metapher handelt. Es geht nicht um Analogie, sondern um unmittelbaren Kontakt zwischen sich erinnernden Gedächtnisträgern und den diese Erinnerung auslösenden, stützenden Merkzeichen der Kultur.

Das Phänomen der menschlichen Zweizeitigkeit oder des doppelten Zeithorizonts tritt uns in den schriftlosen Kulturen mit besonderer Deutlichkeit entgegen. Der Ethnologe Jan Vansina hat den Begriff des floating gap geprägt, um den Unterschied zwischen den beiden Zeithorizonten zu bezeichnen. ${ }^{6}$ „Das Geschichtsbewußtsein“, schreibt er, „kennt nur zwei Register: Zeit der Ursprünge und neuere Zeit. Weil sich die Grenze der Erinnerung mit dem Wechsel der Generationen verschiebt, habe ich die Lücke zwischen beiden „the floating gap“, die fließende Lücke, genannt. Für die Tio im Kongo lag sie im Jahre 1860 ungefähr bei 1800, während sie sich im Jahre 1960 nach ca. 1880 bewegt hatte. "7 Wenn man die Angehörigen einer schriftlosen Stammeskultur nach der Vergangenheit befragt, erhält man in der Regel reiche Auskünfte über eine bis ca. 80 Jahre zurückliegende Zeit, d.h. den 3-Generationenraum des kommunikativen Gedächtnisses. Vergleichbar reich fließen die Auskünfte über eine weit zurückliegende Vergangenheit, in der die Ahnen lebten und die Ordnungen der gegenwärtigen Welt gegründet wurden. Dazwischen liegt die fließende Lücke, das floating gap. Die australischen Aborigines nennen die Zeit der Ursprünge „Traumzeit“ (Dreaming); sie versetzen sich in diese Traumzeit während ihrer jährlichen Wanderungen entlang der „song-lines“. ${ }^{8}$ Hier wird also die Landschaft zum Medium des Kulturellen Gedächtnisses. Wir dürfen davon

6 Jan Vansina, Oral Tradition as History, Madison 1985.

7 A.a.O., $23 f$.

8 T.G.H. Strehlow, Totemic Landscapes, London 1970; W.E.H. Stanner, On Aboriginal Religion, Sidney 1966 Neuausg. 1989. 
ausgehen, daß das Leben in zwei Zeithorizonten, wie es dem Menschen durch das kommunikative und das kulturelle Gedächtnis vermittelt wird, zur Grundausstattung des Menschen gehört. Der Gebrauch der Schrift hat die Grenze zwischen beiden Gedächtnisformen, das floating gap, zunehmend überbrückt. In einer schriftlosen Kultur bieten die Feste und Riten die einzige Form, in der der Einzelne mit dem kulturellen Gedächtnis, der Zeit der Ursprünge in Verbindung treten kann. Die beiden Zeithorizonte gliedern sich hier scharf in Alltagszeit und Festzeit. In einer elaborierten Schriftkultur wie der unsrigen dagegen bietet sich dem Einzelnen jederzeit die Gelegenheit, ein Buch zur Hand zu nehmen, ein Bild zu betrachten, ein Musikstück zu hören, das ihn seinen Alltagsgeschäften entrückt und in einen anderen Zeithorizont versetzt. Entsprechend verblaßt bei uns die Bedeutung der Riten und Feste. In schriftlosen und überhaupt frühen, traditionalen Kulturen bedarf der weitere Zeithorizont der Inszenierung, der „Performanz“, wie man heute sagt. So wie die Vergangenheit uns nur insoweit gegeben ist, als wir uns ihrer erinnern, ist solches Erinnern den frühen Menschen nur so weit möglich, als sie es pflegen und rituell inszenieren. In gewissem Sinne gilt das bis heute. Das kulturelle Gedächtnis bedarf der stützenden Rahmen und Institutionen wie Bibliotheken, Museen, Schulen, Theater, Konzertsäle, Orchester, Kirchen, Synagogen, Moscheen, Lehrer und Bibliothekare, Pfarrer, Rabbis und Scheichs oder Mullahs. Ohne Institutionen, Medien und Spezialisten ist ein kulturelles Gedächtnis nicht möglich. Ein kulturelles Gedächtnis, das uns das Leben in zwei Zeiten ermöglicht, bedarf unablässiger Pflege. Deshalb ist es auch manipulierbar und zerstörbar.

In seinem Roman 1984 hat George Orwell das Schreckbild einer Gesellschaft gezeichnet, die nur noch in einer einzigen Zeit, der von der Partei verordneten „ewigen Gegenwart“ lebt. Durch systematische Spurenverwischung hat es die Partei geschafft, das kulturelle Gedächtnis der Gesellschaft vollständig zu vernichten. Niemand, auch nicht die Alten, die lange vor der „Großen Revolution“ geboren wurden, kann sich mehr daran erinnern, ob die Vergangenheit irgendwie anders, vielleicht gar besser war als die Gegenwart. Das kommunikative Gedächtnis, auf das die Menschen reduziert sind und das voll und ganz von der Partei kontrolliert wird, ist zu jeder Art von kritischer Distanznahme außerstande. Orwells Roman verarbeitet seine Erfahrungen mit dem Stalinismus und Faschismus; es handelt sich hier um eine Art fiktionaler Hochrechnung von Tendenzen, die es immer wieder in der Geschichte gegeben hat und die in den totalitären 
Regimes des 20. Jahrhunderts kulminierten. Das kulturelle Gedächtnis ist nicht nur die tragende, legitimierende Unterfütterung einer jeweiligen $\mathrm{Ge}$ genwart, sondern kann durchaus auch eine kritische, ja geradezu subversive und revolutionäre Instanz darstellen.

Machen wir uns einmal die unterschiedlichen Zeithorizonte oder Verfallsdaten der verschiedenen Gedächtnisformen bzw. -dimensionen klar: Das persönliche Gedächtnis verschwindet mit dem Tod oder dem Gedächtnisverlust des Individuums. Das kommunikative Gedächtnis umfaßt typischerweise einen Zeithorizont dreier miteinander interagierender Generationen, also maximal 80-100 Jahre. Das lateinische Saeculum bestimmte sich nach der Lebensspanne der letzten Zeitzeugen eines Ereignisses. Noch leben einzelne Menschen, die Erinnerungen an den ersten Weltkrieg bewahren; der deutsch-französische Krieg von 1870/71 oder der amerikanische Bürgerkrieg von 1861-65 aber gehören, wie man sagt, der Geschichte und nicht mehr dem kommunikativen Gedächtnis an, weil es keine Zeitzeugen mehr gibt. Halbwachs zog hier, zwischen mémoire und bistoire, eine scharfe Grenze. ${ }^{10}$ In Wirklichkeit aber verlassen wir hier keineswegs den Raum der Erinnerung, sondern gehen vom kommunikativen ins kulturelle Gedächtnis über. Die Vergangenheit ist jenseits des Horizonts lebendiger, in Zeitzeugen verkörperter Erinnerung, keineswegs nur noch „tot“ und Sache objektiver historischer Forschung. Die Schlacht auf dem Amselfeld fand im Jahre 1389 statt. Die Serben haben aber diese Niederlage gegen die Türken in Form mündlich überlieferter Heldenlieder und sogar ihres Heiligenkalenders zum Zentrum ihres kulturellen Gedächtnisses und ihrer politischen Mythologie gemacht, die ihr politisches Handeln noch in den 90er Jahren des 20. Jahrhunderts auf unheilvollste Weise bestimmte. So wie das Jahr 1389 den Serben, ist den Schiiten und Alleviten der 10. Muharram 680 mit Flammenschrift ins Herz geschrieben. An diesem Tag wurde Hussein, der Enkel des Propheten, bei der Schlacht von Kerbela in einen Hinterhalt gelockt und umgebracht; fast die ganze Familie des Propheten wurde aus-

9 B. Gladigow, „Aetas, Aevum und Saeclorum Ordo. Zur Struktur zeitlicher Ordnungssysteme", in: David Hellholm (Hg.), Apocalypticism in the Mediterranean World and in the Near East, Tübingen 1983, 255-271.

10 S. hierzu Verf., Das kulturelle Gedächtnis. Schrift, Erinnerung und politische Identität in frühen Hochkulturen, München 1992, 42-45. Zur Unterscheidung von Geschichte und Gedächtnis bei Pierre Nora und Yosef Hayim Yerushalmi im Anschluß an Maurice Halbwachs s. zahlreiche Beiträge in Michael Brenner, David N. Myers (Hg.), Jüdische Geschichtsschreibung heute. Themen, Positionen, Kontroversen, München 2002. 
gelöscht. Das Aschura-Fest bei dem sich jährlich viele Millionen Schiiten blutig geißeln, hält die Erinnerung an diese Leidensgeschichte lebendig, so wie für die Christen der Karfreitag die Passion Christi in Erinnerung ruft. Die Juden gedenken jährlich in der Seder-Nacht des Auszugs aus Ägypten, der nach biblischer Chronologie im 15. Jh. v. Chr. stattfand. Der typische Horizont kultureller Tiefenzeit umfasst jene 3000 Jahre, auf die Goethe das kulturelle Gedächtnis bezifferte: „Wer nicht von dreitausend Jahren / Sich weiß Rechenschaft zu geben / Bleib im Leben unerfahren, / Mag von Tag zu Tage leben." Die Stadt Jerusalem, die sich auf die Gründung König Davids zurückführt, feierte im Jahre 1996 ihr 3000 jähriges Jubiläum. Der Staat Georgien, der sich auf das aus der Argonautensage bekannte Kolchis zurückführt, feierte sein 3000jähriges Bestehen im Jahre 2000. Das alles hat nichts mit historischer Forschung und mit einem objektiven Interesse für die Vergangenheit zu tun, sowohl Jerusalem als auch Kolchis sind viel älter, sondern allein mit einem menschlichen Grundbedürfnis nach Orientierung in der Zeit, das aufs Engste mit Fragen kollektiver und kultureller Identität und Zusammengehörigkeit zusammenhängt. Im Begriff der Identität, um die es hier geht, ist die Zeit eingeschlossen, denn es geht um das Problem, im Wandel der Zeit derselbe zu bleiben. Sich in der Zeit orientieren heißt, ein Bewußtsein seiner selbst ausbilden, sich denken, erfinden, entwerfen zu können.

In jedem Falle möchte ich, diesen ersten Teil abschließend, festhalten, daß die Konstruktion einer kulturellen Tiefenzeit in engstem Zusammenhang steht mit der Ausbildung einer kulturellen Identität. Diese scheint in zwei Richtungen zu wirken: in der bisher betrachteten Richtung der $\mathrm{Zu}-$ sammengehörigkeit und der Ausbildung von Wir-Bewußtsein, aber auch in der Richtung persönlicher Unsterblichkeit. Ich könnte mir vorstellen, daß dieses Bedürfnis nach kultureller Langzeitorientierung mit so etwas wie einem mehr oder weniger unbewußten Wunsch nach Unsterblichkeit zusammenhängt. Der Mensch ist bekanntlich das einzige Lebewesen, das um seine Endlichkeit und Vergänglichkeit weiß. Auch das verdankt er seinem Gedächtnis und seiner Fähigkeit, sich in der Zeit zu orientieren. Das Kulturelle Gedächtnis erschließt uns jenseits des Horizonts unserer Lebenszeit einen Raum der Unsterblichkeit, den wir vermutlich brauchen, um mit dem Wissen um die Begrenztheit unseres Lebens zurecht zu kommen. Gerade weil wir dank unseres Gedächtnisses unter allen Lebewesen die einzigen sind, die vor unsere Geburt zurück und über unseren Tod hinaus denken und der Begrenztheit unserer Existenz innewerden können, bilden wir ein 
Langzeitgedächtnis aus, in dem unser Vorstellungstrieb und unsere Sehnsucht nach Unsterblichkeit zusammenwirken, um uns jenseits des Kurzzeitgedächtnisses unseres Alltagshandelns einen Raum der Abstandnahme, Besinnung, Reorientierung in der kulturellen Tiefenzeit zu erschließen.

\section{Zyklische und lineare Zeit: Die altägyptische Lehre der „Zwei Ewigkeiten“}

\subsection{Neheh, die,Erneuerungszeit‘ der Riten}

Die Unterscheidung von reversibler und irreversibler bzw. zyklischer und linearer Zeit möchte ich nun nicht theoretisch entfalten, sondern am konkreten Beispiel der altägyptischen Zeitvorstellung illustrieren. ${ }^{11}$ Die Ägypter unterscheiden eine zyklische und eine nicht-zyklische Zeit; die eine nennen sie Neheh, die andere Djet. Neheh, die zyklische Zeit, ist die ewige Wiederkehr des Gleichen; sie wird erzeugt durch die Bewegung der Gestirne und daher mit der Sonne determiniert. Diese Zeit wird mit dem Begriff des Werdens assoziiert, der im Ägyptischen mit dem Bild des Skarabäus geschrieben wird. Der Skarabäus ist bekanntlich das zentrale Heilssymbol im Ägyptischen. Nicht das Sein, sondern das Werden steht im Zentrum ihres Denkens. Die Zyklen werden und vergehen, und was innerhalb der Zyklen wird, vergeht in der Hoffnung erneuerten Werdens. Wir können diese Zeit daher mit vollem Recht als „Erneuerungszeit“ bezeichnen.

Die andere Zeit wird mit dem Begriff des Bleibens, Währens, Dauerns assoziiert. Sie wird mit dem Zeichen der Erde determiniert. Ihre Symbole sind Stein und Mumie, ihr Gott ist Osiris, der gestorbene Gott, der dem Totenreich vorsteht. Djet ist ein heiliger Raum der Dauer, worin das Gewordene, zur Endgestalt Ausgereifte und in diesem Sinne Vollendete unwandelbar fortdauernd aufgehoben ist. Dies genau ist die Bedeutung des Namens, den Osiris als Herr der Djet trägt: Wannafre (gr. Onnophrios)

$11 \mathrm{Zu}$ den Einzelheiten s. Verf., Zeit und Ewigkeit im Alten Ägypten. Ein Beitrag zur Geschichte der Ewigkeit, Heidelberg 1975; „Das Doppelgesicht der Zeit im altägyptischen Denken“, in: A. Mohler, A. Peisl (Hrsg.), Die Zeit (Schriften der C.F. v. Siemens-Stiftung Nr. 6) München 1983, 189-223, wiederabgedr. in: Stein und Zeit. Mensch und Gesellschaft im Alten Ägypten, München 1991, 32-58; „La notion d'éternité dans l'Égypte ancienne“, in: Vinciane Pirenne-Delforge, Öhnan Tunca (Hgg.), Représentations du temps dans les religions, Bibliothèque de la Faculté de Philosophie et Lettres de l'Université de Liège, fasc, CCLXXXVI, Liege 2003, 111-122. 
bedeutet: „Der in Vollendung Währende“. Es handelt sich bei der Djet also nicht um einen linearen Zeitbegriff, sondern vielmehr um dessen Gegenteil und Aufhebung, wobei hier aber nicht der Kreis, sondern der Raum das Gegenteil der Linie bildet. Beide Zeiten oder Ewigkeiten gehören daher als Ausblendung von Wandel und Veränderung auf die Seite des Sakralen. Neheh sakralisiert die Zeit als Bewegung, indem sie zur Kreisbahn und ständigen Erneuerung gebogen wird, Djet sakralisiert die Zeit als währende Dauer, indem sie durch die symbolischen Formen des Steinernen in einen Raum der Unwandelbarkeit geformt wird. Diese Zeit können wir, worauf ich noch eingehen werde, als „Rechenschaftszeit“ bezeichnen. Wir fassen zusammen: Die Erneuerungszeit ist zyklisch und arbeitet durch Ritualisierung gegen das Lineare, Irreversible an, die Rechenschaftszeit ist räumlich ${ }^{12}$ und arbeitet im Medium der Monumente und ihrer streng kanonisierten Formensprache ebenfalls gegen das Irreversible, gegen Veränderung, Verschwinden und Vergessen an.

Der Kult dient in Ägypten in allererster Linie der Konstruktion und InGang-Haltung der Neheh-Zeit, er hat den Charakter eines ritualisierten Kalenders. Wie in vielen anderen Kulturen auch, beobachteten in Ägypten Spezialisten unablässig den Himmel. Während es aber etwa in China, Mesopotamien, Rom und anderswo um die Wahrsagekunst und damit um die Erkennung und Deutung auffälliger Besonderheiten im Sinne von Zeichen ging, galt in Ägypten die auf den Kosmos gerichtete Aufmerksamkeit nicht den Ausnahmen, sondern den Regeln. In der zyklischen Regelhaftigkeit

12 Auf die Raumassoziationen der Djet hat besonders nachdrücklich Wolfhart Westendorf hingewiesen: „Raum und Zeit als Entsprechung der beiden Ewigkeiten“, in Fontes atque Pontes. Festgabe Hellmut Brunner, Wiesbaden 1983, 422-435. Westendorf vertritt in diesem Aufsatz die Ansicht, bei Neheh und Djet handele es sich geradezu um die ägyptischen Äquivalente von „Zeit“ und „Raum“. In der Tat schreibt W. Kaempfer: „Was wir Raum nennen, ist insofern nichts als die Verwirklichung von Zeit, und was wir Zeit nennen, reine Potentialität" (Kaempfer, Die Zeit und die Uhren, Frankfurt 1991, 18). In genau diesem Sinne läßt sich auch die Unterscheidung von Djet und Neheh verstehen, die ich in meiner 1975 erschienenen Monographie als „aktualisierte“ und „virtuelle Zeit“ gegenübergestellt hatte. Djet ist aktualisierte und insofern verräumlichte Zeit. Als solche kann sie dem allwissenden Gott „vor Augen stehen“, vgl. den Vers „Die Djet steht ihm vor Augen wie der gestrige Tag, wenn er vergangen ist", die an Ps. 90.4 erinnert und in zwei ägyptischen Hymnen begegnet (Zeit und Ewigkeit, 69). Djet ist „,der Raum der Dauer“ bzw. „die Dauer des Raumes“, aber wohl kaum der reine physikalische Begriff des Raumes (den es im Ägyptischen ebensowenig gibt wie den reinen physikalischen Begriff der Zeit). 
seiner Prozesse offenbarte sich dem Ägypter die Göttlichkeit des Kosmos. Die Ägypter beobachteten den Himmel nicht, um die Zukunft vorherzusagen, sondern um die Zeit als solche in Gang zu halten. Sie stellten die Stunden, Tage, Monate und Jahre fest. Der ägyptische Mond- und Festkalender beruht auf Beobachtung. Anbruch und Länge des Mondmonats, der bekanntlich zwischen 29 und 30 Tagen schwankt, wurde nicht durch Berechnung, sondern durch Beobachtung bestimmt. Auch der Anfang des Jahres beruht auf astronomischer Beobachtung (Frühaufgang des Sirius). Da man aber für andere Zwecke auch einen auf Berechnung beruhenden Kalender braucht, arbeiteten die Ägypter mit zwei Kalendern.

Der ägyptische Kalender beruhte auf dem Sonnenjahr, das mit $365 \mathrm{Ta}-$ gen, also um einen Vierteltag zu kurz, berechnet wurde. Es begann mit dem Einsetzen der Nilüberschwemmung, das mit einem astronomischen Ereignis, dem Frühaufgang der Sothis (=Sirius) verknüpft wurde (Mitte Juli nach dem julianischen Kalender) und gliederte sich in 12 Monate zu je $30 \mathrm{Ta}-$ gen, die zu drei Jahreszeiten zusammengefaßt wurden. Am Ende dieser 360 Tage wurden 5 „Zusatztage“ (Epagomenen) angehängt. Die Monatsnamen sind von Festnamen abgeleitet; der Kalender ist also ein Festkalender. Wie in allen archaischen und traditionalen Kulturen haben Zeitmessung und Kalender auch in Ägypten ihren Sitz im Kult, der in weit höherem Maße als die zivilen Geschäfte eine präzise Ordnung der Zeit erforderte.

Das Mondjahr bestand ebenfalls aus 12 Monaten, die sich zu 354 Tagen addierten, was alle drei, seltener zwei Jahre die Einfügung eines Schaltmonats erforderte, um mit dem Jahreszyklus in Einklang zu bleiben. Die Ägypter hielten neben dem Normaljahr an diesem Mondjahr fest, benutzten also zwei Kalender gleichzeitig. Der eine bot den Vorteil der Berechenbarkeit, da er nicht auf Beobachtung beruhte und war daher besonders geeignet für die Zwecke der Verwaltung. Der andere bot den Vorteil des Einklangs mit kosmischen Zyklen und war daher besonders geeignet für die Zwecke des Kults, dem es auf die Eingliederung der menschlichen in die als göttlich verehrten natürlichen Ordnungen ankam. Trotzdem darf man die beiden Kalender nicht als „zivil“ und „religiös“ verstehen. Beide Kalender sind religiös. Der Normalkalender ist sogar auch in dieser Hinsicht noch bedeutungsvoller als der Mondkalender, weil die nach ihm berechneten Feste, die „Feste auf dem Jahr“, viel wichtiger waren als die nach dem Mondkalender beobachteten „Feste des Himmels“. Der Kalender ist als solcher religiös, weil die Ordnung als solche heilig ist. Die Ägypter haben die beiden Kalender durch Konkordanzen miteinander in Beziehung gesetzt. Dabei haben 
sie einen Zyklus von 25 Normalkalenderjahren zugrundegelegt, die genau 309 Mondmonaten entsprechen. Eine solche Konkordanz ist in einem Papyrus der Spätzeit erhalten.

Ich möchte die ägyptische Form des Kalenders als eines Instruments der Zyklisierung und In-Gang-Haltung der Zeit an zwei Beispielen illustrieren, die mir als besonders typisch erscheinen. Das erste Beispiel ist das Stundenritual, das zweite die Tagewählerei. Das Stundenritual ist ein in allen Sonnenheiligtümern des Landes durchgeführtes Ritual stündlicher Lobpreisungen des Sonnenlaufs. Dabei geht es darum, den Sonnengott und die bei diesem Vorgang beteiligten Götter in ihrem unausgesetzten Kampf gegen die Gravitation in Richtung Stillstand und Aufösung zu unterstützen. Ohne solche Bemühung würde die Sonne nicht weiterkommen, zumindest ein bestimmter, prozeßhaft gedachter Sinnzusammenhang abreißen. Dieser Sinnzusammenhang muß rund um die Uhr hergestellt bzw. aufrechterhalten werden. Dem dienen alle Rituale, aber das Stundenritual bringt diesen Sinn der Rituale besonders deutlich zum Ausdruck. Die Wendung „rund um die Uhr“ hat hier einen besonders prägnanten Sinn. Ohne das Ritual würde die Uhr stehenbleiben.

Das zweite Beispiel sind die Tagewählereikalender oder Hemerologien. ${ }^{13}$ Sie sind für Ägypten so typisch, daß im europäischen „Aberglauben“ bis weit in die Neuzeit hinein und möglicherweise in abgeschiedenen Gegenden bis heute bestimmte Unglückstage als „ägyptische Tage“ bezeichnet wurden. ${ }^{14}$ Dieser Kalender verband jeden einzelnen Tag des Jahres mit einem mythischen Ereignis, das ihm eine bestimmte Bedeutung und eine Eigenschaft verlieh. Es gab drei Qualitäten: gut, unbestimmt und unheilvoll. Jeder Tag war in drei Tageszeiten unterteilt, und jede dieser drei Tageszeiten ließ sich aufgrund der dem Tag spezifischen mythischen Episode als gut, unbestimmt oder unheilvoll qualifizieren. Die hemerologische Semiotik liegt ein für alle Mal fest; die Hemerologien sind immerwährende Kalender. Auf einmalige, unvorhergesehene und kontingente Ereignisse - also auf das, was wir unter Geschichte verstehen würden - sind sie nicht eingerichtet. Sinnvoll, bedeutungsvoll und daher mit Zeichencharakter ausgestattet ist nur, was sich kalendarisch wiederholt. Es handelt sich also um das genaue Gegenteil von Prodigien, Wunderzeichen, Vorzeichen usw., die sich ja gerade dadurch über-

13 Christian Leitz, Tagewäblerei. Das Buch h3t nhb ph.wy dt und verwandte Texte, Ägyptologische Abhandlungen 55, Wiesbaden 1994.

14 Emma Brunner-Traut, „Mythos im Alltag. Zum Loskalender im alten Ägypten“, in: Antaios XII, 1971, 332-347 
haupt erst als Zeichen konstituieren, daß sie aus dem Rahmen fallen. Was in Ägypten aus dem Rahmen fällt, verliert seinen Sinn und damit seinen Zeichencharakter. Die kalendarische Zeit ist also in Ägypten keine Leerform, in die die Ereignisse einströmen, sondern eine prall mit Sinn erfüllte Form, die unablässig rituell aufgeführt wird, um den Zustrom der Ereignisse abzuwehren. Was sich dann trotzdem ereignet, und was wir „Geschichte“ nennen würden, hat sich diesem Rahmen einzufügen.

Man kann diese ägyptische Zeitstrategie „Gegenwartsbewältigung“ nennen. Gegenwartsbewältigung richtet sich gegen die Angst, die von Bruch, Wandel und Veränderung ausgeht. Die Gegenwart wird bewältigt, indem jeder Tag in eine heilige Ordnung eingefügt wird, die nur bewahrt, beachtet und begangen werden muß, um als Medium der Zeitbewältigung zu funktionieren. Weil es hier in erster Linie um Gegenwartsbewältigung geht, sucht man vergebens nach größeren retrospektiven Rekonstruktionen von Vergangenheit. Aufgezeichnet werden nur einzelne Ereignisse. Bei dem, was als aufzeichnungswürdig festgehalten wird, wird keine scharfe Grenze gezogen zwischen dem Feiern von Festen, dem Errichten von Bauwerken und dem Erringen von Siegen. Alles schreibt sich ein in die Form des Kalenders, die das Gelingen von Ordnung unter den Bedingungen der Zeitlichkeit vorschreibt, indem sie die Zeit rhythmisiert, Kontinuität stiftet, Bruch, Wandel und Veränderung ausschließt und einen Sinnhorizont ausleuchtet, innerhalb dessen man sich orientieren und identifizieren kann. ${ }^{15}$

Die ägyptische Formel für die Konzeption einer sich zyklisch erneuernden Zeit ist „Tag für Tag wie beim Ersten Mal“. ${ }^{16}$ Das „Erste Mal“ ist der ägyptische Begriff für „Schöpfung“, genesis, bereschit. Mit jedem Tag wiederholt sich das Erste Mal, mit jedem Zyklusende taucht die kreisende Zeit zurück in die vorweltliche Zeitlosigkeit. Eine als Nutbuch bekannte Kosmologie beschreibt den Sonnenaufgang folgendermaßen:

Er (der Sonnengott) entsteht wie er entstand beim Ersten Mal in der Erde des Ersten Males. ${ }^{17}$

15 E. Hornung hat für dieses Geschichtsbild die glückliche Formel „Geschichte als Fest" geprägt: Geschichte als Fest. Zwei Vorträge zum Geschichtsbild der frühen Menschheit, Darmstadt 1966.

16 Siehe hierzu auch P. Vernus, Essai sur la conscience de l'histoire dans l'Égypte pharaonique, Paris 1996, 36-42.

17 O. Neugebauer/R. Parker, Egyptian Astronomical Texts I, Brown Egyptological Studies, Providence 1960, Tf. 44 Text H. 
An jedem Abend stirbt der Sonnengott; das bedeutet das Ende des Tageszyklus, aber es bedeutet keine Katastrophe, weil der Gott, wie es in den Texten immer wieder betont wird, in die Erde eingeht, aus der er entstanden ist, und in den Mutterschoß, aus dem er hervortrat. Sein Weg rundet sich also zur vollendeten Kreisbahn, und dieser Tod mündet notwendigerweise in eine neue Geburt und ist nur der Übergang zu einem neuen Zyklus. Der Tod ist nicht Ende, sondern Übergang, aber nur unter der Bedingung, daß dieses Ende in irgendeiner geheimnisvollen Weise mit dem Anfang zur Deckung kommt und sich die Lebensbahn der Sonne zur Kreisbahn schließt. Dieser Vorgang bildet die ägyptische Heilsgeschichte. Im Mysterium des allnächtlichen Sonnentodes und der allmorgendlichen Neugeburt gründen alle Jenseits- und Unsterblichkeitshoffnungen des Ägypters. ${ }^{18}$ Deshalb, aufgrund dieser Analogie, ist der Vollzug der Riten und die rituelle Formung der Zeit so ungeheuer wichtig. So wie der Kosmos und die Zeit im Sonnenlauf, so soll sich auch die menschliche Welt in ihren staatlichen, gesellschaftlichen und persönlichen Abläufen immer wieder erneuern und in den Idealzustand des „Ersten Males“ zurückkehren. Denn die zyklische Zeit, die auf diese Weise erzeugt wird, ist eine gewissermaßen heilswirksame, bedeutungsvolle Zeit, in der auch der Mensch auf Erneuerung hoffen darf. Er hat an denselben Riten und Deutungen Anteil. So wie der Sonnenuntergang in den Deutungsmustern des Lebensendes, so erscheint das Lebensende in den Deutungsmustern des Sonnenuntergangs - die beiden Ereignisse beleuchten sich gegenseitig. Gelingen bedeutet in ägyptischer Vorstellung nicht Fortschritt, sondern Rückkehr: Rückkehr zum Ersten Mal, zu den Vorbildern der Vergangenheit, zu den Normen der Vorfahren.

\subsection{Djet: die ,Rechenschaftszeit' der Monumente}

Abschließend möchte ich noch einen Blick auf die andere Zeit werfen: Djet, die Zeit der unwandelbaren Fortdauer und zugleich, das ist die These, die moralische Zeit, die Zeit der Rechenschaft und der Verantwortung. Djet ist die Zeit des Steins. Mit diesem Aspekt der Zeit verlassen wir das Reich des Sonnengottes und betreten das Reich des Osiris, des Gottes der Toten, der selbst ein Toter ist und als solcher in unwandelbarer Vollkommenheit fortdauert. Das bringt auch sein Name zum Ausdruck: Wannafre, griechisch Onnophrios, heißt: der in Vollendung Existierende. So wie die

18 S. hierzu Verf., Tod und Jenseits im Alten Ägypten, München 2001, Kap. VII: „Der Tod als Heimkehr". 
Neheh-Zeit mit dem Sonnengott und dem Werden, so ist die Djet-Zeit mit Osiris und dem Sein verbunden.

Die moralische Konstruktion der Zeit beruht auf dem Gedanken, daß man für sein Handeln und Unterlassen zur Rechenschaft gezogen wird. Zwischen „Tun“ und „Ergehen“ gibt es einen, wie immer verborgenen, $\mathrm{Zu}$ sammenhang. Nichts bleibt folgenlos, irgendwann und irgendwie rächt sich das Böse und lohnt sich das Gute. Die Ägypter denken sich den Tun-Ergehen-Zusammenhang aber nicht als einen verborgenen Automatismus, eine Art Naturgesetz, sondern als eine Ordnung, die sie selbst herstellen und aufrecht erhalten müssen. Diese Ordnung heißt ägyptisch Ma'at, ein Wort, das wir mit Begriffen wie Ordnung, Gerechtigkeit, Wahrheit umschreiben, aber nicht eigentlich übersetzen können. Dazu ließe sich sehr viel sagen, ich möchte mich hier aber auf den einen Punkt der Zeit, und zwar der moralischen Konstruktion der Zeit beschränken. In dieser Konstruktion erscheint die Zeit als ein Zusammenhang von Tat und Folge, der durch die Ma'at garantiert wird. Die Menschen müssen die Ma'at in ihrer Lebensführung verwirklichen und zwar dadurch, daß sie aneinander denken und füreinander handeln. Ma'at ist der Zentralbegriff einer im höchsten Maße kooperativen Ethik. Der Tun-Ergehen-Zusammenhang ist eine Sache des Gedächtnisses und der Aufmerksamkeit füreinander. Ich habe dafür den Begriff einer iustitia connectiva vorgeschlagen, einer verbindenden Gerechtigkeit.

In der Ausformulierung dieses Prinzips des Aneinander-Denkens und Füreinander-Handelns kommen die Ägypter bereits erstaunlich nah an Gedankengänge heran, die Nietzsche in seiner Genealogie der Moral entwickelt hat. Für Nietzsche ist der Mensch, „das Tier, das versprechen kann“: er kann sich auf Zukunft festlegen und daher für seine Taten verantwortlich gemacht werden. Dieses Gedächtnis ist es, was nach Auffassung der Ägypter den Tun-Ergehen-Zusammenhang in Kraft setzt. Er wirkt, wie gesagt, nicht automatisch wie ein Naturgesetz, sondern nur im Raum der gegenseitigen Erinnerung und Aufmerksamkeit, des Aneinander-Denkens und Füreinander-Handelns. Sinn oder Nicht-Sinn der Welt ist, mit anderen Worten, eine Frage des Eingedenkseins oder der Vergessenheit. Wir können die moralische Konstruktion der Zeit daher als Rechenschafts- und Gedächtniszeit bezeichnen.

Gedächtnis, Gewissen, Verantwortung: das alles konstituiert nun auch sehr zentral den ägyptischen Begriff der Gerechtigkeit als Inbegriff einer Lebensführung, die dem Einzelnen unvergängliche Fortdauer im Gedächt- 
nis der Nachwelt verheißt. Gerechtigkeit ist nach ägyptischer Auffassung ein moralisches Gedächtnis, das dem einzelnen Leben Kontinuität und Identität gibt und dafür sorgt, ganz im Sinne von Nietzsches „berechenbaren Individuum“, daß man morgen derselbe ist wie heute. ${ }^{19}$ Die Ägypter orientieren sich genau wie die Babylonier so in der Zeit, daß sie die Vergangenheit vor Augen und die Zukunft im Rücken haben. Das Frühere ist „Vorn“, das Zukünftige ist „hinten“ oder „im Rücken“. Daher entwickeln sie diese Zusammenhänge nicht wie Nietzsche am Paradigma des Versprechens, der Obligation, der Verpflichtung auf Zukunft, sondern am Paradigma der Dankbarkeit, der Verantwortung, der Verpflichtung gegenüber der Vergangenheit. In einem Text aus dem frühen 2. Jahrtausend heißt es: „Der Träge hat kein Gestern“20, d.h. kein Gedächtnis, kein Gewissen, keine Verantwortlichkeit, keine Vergangenheit. Der verantwortliche Mensch kehrt, wie es im gleichen Text heißt, „zurück an seine Stelle von gestern, denn es ist befohlen: Handle für den, der handelt, um zu veranlassen, daß er tätig bleibt. Das heißt, ihm danken für das, was er getan hat “21. Wenn die Vergangenheit - das „Gestern“ - vergessen wird, geht der Tun-Ergehen-Zusammenhang, die moralische Konstruktion der Zeit aus den Fugen. Das wird in vielen ägyptischen Texten beklagt, die ich hier aber nicht weiter anführen möchte. Diese Texte machen den Zusammenhang von Gedächtnis und Gerechtigkeit deutlich und damit den Appellcharakter der Rechenschaftszeit als eines moralischen Imperativs.

Die Ägypter dachten sich, daß sie nach dem Tode vor Osiris und einem Totengericht Rechenschaft ablegen müßten für ihre Lebensführung. Während sie beteuerten, der Ma'at gemäß gelebt und eine lange Liste von Sünden nicht begangen zu heben, wurde ihr Herz auf eine Waage gelegt. Mit jeder Lüge würde es schwerer werden und dann von dem Monstrum, das neben der Waage sitzt verschlungen werden. Wer aber ohne Sünde befunden wurde, der wurde in das Reich des Osiris und in die Zeit ewiger Fortdauer aufgenommen. ${ }^{22}$ Sie wollten aber nicht nur im Reich des Osiris, sondern auch auf Erden fortdauern, im Gedächtnis der Nachwelt. Daher errichteten sie sich, sofern sie sich das irgend leisten konnten, steinerne Grabmo-

19 Vgl. zum Folgenden Verf., Ma'at. Gerechtigkeit und Unsterblichkeit im alten Ägypten, München 1990, 60-64.

20 Bauer B 2, 109f.; Verf., a.a.O., 60.

21 Bauer B 1, 109-110; Friedrich Vogelsang, „Kommentar zu den Klagen des Bauern“, Unters. z. Gesch. u. Altertumsk. Äg. 6, Leipzig 1913, S. 100.

22 S. hierzu Verf. Tod und Jenseits, 100-115. 
numente, um im Medium des Steins in die Djet-Zeit der unwandelbaren Fortdauer einzugehen. In diese Gräber schrieben sie ihre moralischen Leistungen, ihren Lebensbeitrag zur Verwirklichung der Ma'at, durch Aneinander-Denken und Füreinander-Handeln, damit auch an sie gedacht und für sie gehandelt würde. Daher ist das ägyptische Monumentalgrab nicht nur ein Medium des Gedächtnisses, sondern auch, mit Schiller zu reden, eine "moralische Anstalt".23

Das haben noch die Griechen verstanden. Hekataios von Abdera, der von 320 bis 305, also 15 Jahre lang, in Alexandrien lebte, schreibt:

Die Einheimischen geben der im Leben verbrachten Zeit einen ganz geringen Wert. Dagegen legen sie das größte Gewicht auf die Zeit nach ihrem Tode, während der man durch die Erinnerung an die Tugend im Gedächtnis berwahrt wird. Die Behausungen der Lebenden nennen sie „Absteigen“ (katalyseis), da wir nur kurze Zeit in ihnen wohnten. Die Gräber der Verstorbenen bezeichnen sie als „ewige Häuser“ (aidioi oikoi), da sie die unendliche Zeit im Hades verbrächten. Entsprechend verwenden sie wenig Gedanken auf die Ausrüstung ihrer Häuser, wohingegen ihnen für die Gräber kein Aufwand zu hoch erscheint. ${ }^{24}$

Hekataios stellt in diesen Sätzen zweierlei fest: daß die eigentlich bedeutsame Zeit für die Ägypter die Zeit nach dem Tode ist, und daß diese Dauerzeit durch Gedächtnis und Gerechtigkeit erzielt wird. Man wird unsterblich, weil man auf Grund seiner Tugend im Gedächtnis der Menschen fortdauert, und diese Tugend besteht ihrerseits in einem moralischen Gedächtnis, das den Menschen gesellschaftsfähig macht, indem es ihn seiner Verpflichtungen eingedenk sein und morgen derselbe wie gestern und heute sein läßt. Noch einmal bekräftigt ein Grieche, über 2000 Jahre nach Errichtung der Pyramiden, die unaufösliche Allianz zwischen Stein, Moral und Ewigkeit, die sich mit dem Begriff der Djet als der „moralische Zeit“ verbindet.

\subsection{Die Verknüpfung von Neheh und Djet}

Das besondere der ägyptischen Konstruktion der Zeit ist nun aber nicht nur die Unterscheidung, sondern vor allem die Verbindung der beiden Aspekte, Neheh und Djet, die erst zusammen die Zeit ergeben. Die Zeit, wie

23 Verf., Tod und Jenseits, 476-490.

24 Hekataios von Abdera, bei Diodor, Bibl.Hist. I 51; Tod und Jenseits, 483. 
sie die Ägypter erlebten, entstand oder bestand aus einer Komplexion von Neheh und Djet. Darauf will ich abschließend eingehen. Viele Darstellungen des Sonnengottes zeigen ihn, wie er bei Tage den Himmel befährt und bei Nacht Osiris in der Unterwelt bestrahlt. ${ }^{25}$ Diese nächtliche Vereinigung von Re und Osiris haben wir uns als eine Verbindung der Neheh- und der Djetzeit vorzustellen. Im Grab der Nefertari ist eine widderköpfige Mumie dargestellt mit einer Sonnenscheibe auf dem Kopf, die von Isis und Nephthys flankiert und beschützt wird. Die Beischrift links unten erklärt: „das ist Osiris, der in Re ruht“, während die zur Rechten besagt „das ist Re, der in Osiris ruht. “26 In anderen Darstellungen wird diese Figur als Ba Demedj „der vereinigte Ba“ bezeichnet: in ihm vereinigen sich die Ba's, d.h. die „Seelen“ von Re und Osiris. Diese Vereinigung von Re, dem Exponenten der ewig kreisenden kosmischen Zeit, und Osiris, dem Exponenten der unwandelbaren Fortdauer des Vollendeten, ereignet sich in der Mitternacht. Im 17. Kapitel des Totenbuchs findet sich ein weiteres Bild für die Zeit. Zwei nach außen gewendete Löwen flankieren die Hieroglyphe für das Wort „Achet“, das wir mit Horizont übersetzen. Eigentlich bezeichnet es die Stelle, an der, zwischen zwei Bergen, die Sonne auf und untergeht. Beim linken Löwen steht „der morgige Tag“, beim rechten „der gestrige Tag“. ${ }^{27}$ Das ist logisch, denn die Ägypter orientierten sich nach Süden, für sie war also Westen, Sonnenuntergang und Gestern rechts, Osten, Sonnenaufgang und Morgen links. Der Text zu dieser Darstellung lautet:

Ich bin das Gestern, ich kenne das Morgen.

Was bedeutet das?

Was Gestern betrifft: Osiris ist es.

Was Morgen betrifft: Re ist es. ${ }^{28}$

Im gleichen Text heißt es auch:

Was Neheh betrifft, das ist der Tag.

Was Djet betrifft, das ist die Nacht. ${ }^{29}$

25 Z.B. Verf., Tod und Jenseits, 253 Abb. 33.

26 A.a.O., 251 Abb. 32.

27 A.a.O., 250 Abb. 31.

28 E. Hornung, Das Totenbuch der Ägypter, Zürich 1979, 60f.

29 A.a.O., 62. 
In einem Papyrus aus dem 11. Jh. v. Chr. finden wir die Löwen „Gestern“ und „Morgen“ wieder, hier aber nicht mit der Horizont-Hieroglyphe, sondern mit der Sonnenscheibe zwischen ihren Rücken, in der ein Kind sitzt. Die Sonnenscheibe ist von einer Schlange umgeben, die sich in den Schwanz beißt. Die Griechen haben dieser Schlange den Namen Uroboros gegeben und sie als ein Symbol der ewig in sich kreisenden Zeit, also des Neheh gedeutet. ${ }^{30}$ Die beiden Löwen aber, die die Sonnenscheibe mit dem Kind darin flankieren und die für Gestern und Morgen, oder auch Tag und Nacht, und daher für Neheh und Djet stehen, symbolisieren das Ganze der Zeit, das sich aus der Vereinigung dieser beiden Aspekte ergibt.

Aber nicht nur der Kosmos, auch der Mensch lebt in beiden Zeiten zugleich. Das gilt allerdings nur für das ewige Leben, in das er nach ägyptischer Vorstellung nach dem Tode eingeht, wenn er die Prüfung des Totengerichts besteht. Während seines Erdendaseins lebt er in einer anderen Zeit, die auf ägyptisch ahau „Lebenszeit“, oder auch schai „Schicksal“ heißt. Das ist die bemessene Zeit, die allem Irdischen gegeben ist, im Gegensatz zur kosmischen Zeit, in der die Gestirne kreisen und in der Osiris unwandelbar in der Unterwelt ruht. ${ }^{31}$ Hier stoßen wir innerhalb der ägyptischen Zeitkonstruktion auf jene Unterscheidung zwischen Kurzzeit und Langzeit, von der wir ausgegangen sind. Aber auch während seines Erdendaseins, also in der Ahau-Zeit, vermag der ägyptische Mensch mit der kosmischen Zeit in Verbindung zu treten. Im Vollzug der Riten, deren immer gleicher Ablauf die ewige Wiederkehr der kosmischen Zyklen im menschlichen Handeln abbildet, gewinnt er Anteil an der Neheh-Zeit, und durch die Errichtung der Monumente hofft er sich in die Djet-Zeit hineinstellen zu können. Nach dem Tode aber will er ganz in die kosmische Zeit in ihren beiden Aspekten eingehen. Im Balsamierungsritual sagt der Priester zum Toten:

Möge dein Ba existieren, indem er im Neheh lebt wie Orion im Leib der Himmelsgöttin; und indem dein Leichnam dauert in der Djet wie der Stein des Gebirges. ${ }^{32}$

30 Verf., Stein und Zeit, 57 Abb. 4.

31 Verf., Zeit und Erigkeit, 11-18, 45-48.

32 Balsamierungsritual Papyrus Boulaq 3, VIII, 3-4; s. Serge Sauneron, Le rituel de l'embaumement, Kairo 1952, Tf. 27. 
Die kulturelle Zeitkonstruktion der alten Ägypter war, wie wir sehen, ganz besonders stark von dem Wunsch nach Unsterblichkeit geprägt. Unsterblichkeit strebte er an in den beiden Aspekten der Ewigkeit. In der Erneuerungszeit des Neheh wollte er mit dem Sonnengott über den Himmel fahren, und in der Rechenschaftszeit der Djet wollte er unter voller Bewahrung seiner individuellen Identität mit allen Titeln und Würden in die Gemeinschaft des Osiris eingehen. Im Aspekt der Rechenschaftszeit prägt sich auch der Identitätstrieb der Ägypter und das Bedürfnis nach Zugehörigkeit aus. Totengericht und Osiris symbolisieren das Urteil der Mit- und Nachwelt, die erstrebte Unsterblichkeit ist nichts anderes als die Fortdauer im sozialen Gedächtnis der Nachwelt. Diese Fortdauer strebten die Ägypter durch Errichtung steinerner Monumente an, die ihnen zugleich die Evidenz der Djet-Zeit unwandelbarer Fortdauer ständig vor Augen führten. 
